\title{
Flow of a Burger's Fluid in a Channel Induced by Peristaltic Compliant Walls
}

\author{
I. Ahmad, ${ }^{1}$ N. Ali, ${ }^{2}$ A. Abbasi, ${ }^{1}$ W. Aziz, ${ }^{3}$ M. Hussain, ${ }^{1}$ M. Ahmad, ${ }^{1}$ M. Taj, ${ }^{1}$ and Q. Zaman ${ }^{1}$ \\ ${ }^{1}$ Department of Mathematics, University of Azad Jammu \& Kashmir, Muzaffarabad 13100, Pakistan \\ ${ }^{2}$ Faculty of Basic and Applied Sciences, Department of Mathematics and Statistics, International Islamic University, \\ Islamabad 44000, Pakistan \\ ${ }^{3}$ Department of Computer Sciences and Information Technology, University of Azad Jammu \& Kashmir, Muzaffarabad 13100, Pakistan
}

Correspondence should be addressed to I. Ahmad; aaiftikhar@yahoo.com

Received 16 May 2014; Revised 11 August 2014; Accepted 11 August 2014; Published 28 October 2014

Academic Editor: Takeshi Iwamoto

Copyright (C) 2014 I. Ahmad et al. This is an open access article distributed under the Creative Commons Attribution License, which permits unrestricted use, distribution, and reproduction in any medium, provided the original work is properly cited.

A theoretical analysis is presented for the peristaltic motion of a magneto-hydrodynamic (MHD) non-Newtonian fluid in channel with complaint walls. The fluid obeys viscoelastic non-Newtonian model with Burger's constitutive equation. The relevant equations are first developed and then solved using perturbation technique. Expressions of stream function and velocity components are constructed under the assumption that $\delta$ (characteristic ratio of transversal and axial scales of peristaltic motion) is small. The results indicate the strong effects of Burger's fluid parameter, Hartman number, Reynolds number, and complaint wall parameters on the velocity field and stream function. The obtained solutions are shown graphically for the different values of involved parameters.

\section{Introduction}

Peristaltic pumping is a mean of fluid transport caused by waves of contraction along the boundary of a channel/tube. To analyze the properties of peristalsis theoretically, the flow caused by sinusoidal traveling waves of contraction along the boundary has been considered in an infinite channel/tube. In general, flow in a peristaltic channel is driven by two independent mechanisms: the movement of the walls and due to the mean pressure gradient along the channel. After the pioneering work of Shapiro et al. [1] the fluid flow due to prescribed wall motion has been described theoretically by Ebaid [2]. The approach of Fung and Yih [3] was used to analyze the peristaltic flow by Hayat et al. [4] and Haroun [5]. In the above mentioned studies, peristaltic motion was investigated under various assumptions. One of the important aspects in such studies is the nature of the fluid. Since, it is a fact that most of the biological and industrial fluids are non-Newtonian in nature, therefore, peristaltic flow of these fluids has also been studied by Hayat et al. [6] considering non-Newtonian Maxwell fluid in a channel. Srinivas et al. [7] studied the MHD peristaltic motion with slip and heat characteristics. The effects of wall flexibility on the peristaltic motion have also attracted the attention of various researchers in the field. Mittra and Prasad [8] initiated the study of peristaltic transport of viscous fluid with compliant walls. Their work has been generalized by Muthu et al. [9] for viscous incompressible fluid in circular tube and Abd Elnaby and Haroun [10] for two-dimensional channel. The work in [10] has been extended for Maxwell fluid by Ali et al. [11]. Peristaltic motion of Jeffrey fluid in a channel having compliant walls was also studied by Hayat et al. [12]. Recently the heat and mass transfer effects on the peristaltic flow of an Oldroyd-B fluid in a channel with complaint walls were investigated by Hayat et al. [13]. Motivated by the above mentioned investigations, the aim of the present paper is to extend the work of [13] by considering the fluid which obeys the constitutive equation of Burger's fluid model. The Burger's fluid is a rate type viscoelastic fluid model [14] which has been found quite successful in predicting the behavior of viscoelastic material, food products such as cheese and soil. The viscoelastic behavior of fine-grained polycrystalline olivine can also be predicted by Burger's fluid model [15]. Moreover, this model has also been used for calculation of 
transient creep proportion of the earth's mantle [16] and in the interpretation of postglacial uplift [17].

The paper is arranged in the following manner. Flow geometry is explained in Section 2 while governing equations are developed in Section 3. Series solution is provided in Section 4. Results and discussion presented in Section 5. Some conclusions are made at the end of article in Section 6.

\section{Flow Geometry}

Let us consider the flow of magneto hydrodynamic (MHD) Burger's fluid due to sinusoidal motion of the compliant walls in a channel. A constant magnetic field of strength $B_{0}$ is applied in the direction normal to the flow. The deformation of the walls of the channel is represented by the expression

$$
y= \pm \eta(x, t)= \pm\left\{d_{1}+a \sin \frac{2 \pi}{\lambda}(x-c t)\right\}
$$

where $a$ is the amplitude of the peristaltic wave, $\lambda$ is the wavelength, $t$ is the time, $c$ is the speed of the wave, and $d_{1}$ is the half-width of the channel.

\section{Governing Equations}

Let $u$ and $v$ denote the velocity components in the axial and transverse direction, respectively, then we can write

$$
\mathbf{V}=[u(x, y, t), v(x, y, t), 0] \text {. }
$$

The law of conservation of mass and momentum for the flow of an incompressible fluid are

$$
\begin{gathered}
\operatorname{div} \mathbf{V}=0, \\
\rho \frac{d \mathbf{V}}{d t}=-\nabla p+\operatorname{div} \mathbf{S},
\end{gathered}
$$

where $\mathbf{V}$ is the velocity, $\rho$ is the density, $p$ is the pressure, and $\mathbf{S}$ is the extra stress tensor, which satisfies the following relation for a Burger's fluid:

$$
\mathbf{S}+\lambda_{1} \frac{D \mathbf{S}}{D t}+\beta \frac{D^{2} \mathbf{S}}{D t^{2}}=\mu\left(1+\lambda_{2} \frac{D}{D t}\right) \mathbf{A}_{1}
$$

In the above equation $\mu$ is the dynamic viscosity, $\mathbf{A}_{1}$ is the first Rivlin-Ericksen tensor, $\lambda_{1}$ and $\lambda_{2}$ are the relaxation and retardation times, respectively, $\beta$ is Burger's fluid parameter, $\mathbf{L}$ is the gradient of velocity, and $D / D t$ is the upper convective derivative and defined as

$$
\frac{D \mathbf{S}}{D t}=\frac{\partial \mathbf{S}}{\partial t}+(\mathbf{V} \cdot \nabla) \mathbf{S}-\mathbf{L S}-\mathbf{S L}^{T}
$$

Using the definition of velocity and $D / D t$ in (3) we get the following equations:

$$
\begin{gathered}
\frac{\partial u}{\partial x}+\frac{\partial v}{\partial y}=0 \\
\rho\left(\frac{\partial}{\partial t}+u \frac{\partial}{\partial x}+v \frac{\partial}{\partial y}\right) u=-\frac{\partial p}{\partial x}+\frac{\partial S_{x x}}{\partial x}+\frac{\partial S_{x y}}{\partial y}-\sigma B_{0}^{2} u \\
\rho\left(\frac{\partial}{\partial t}+u \frac{\partial}{\partial x}+v \frac{\partial}{\partial y}\right) v=-\frac{\partial p}{\partial y}+\frac{\partial S_{x y}}{\partial x}+\frac{\partial S_{y y}}{\partial y}, \\
\rho \frac{\partial u}{d t}\left(\frac{\partial u}{\partial y}+\frac{\partial v}{\partial x}\right) \quad \\
=\left(\frac{\partial^{2}}{\partial y^{2}}-\frac{\partial^{2}}{\partial x^{2}}\right) S_{x y}+\frac{\partial^{2}}{\partial x \partial y}\left(S_{x x}-S_{y y}\right)-\sigma B_{0}^{2} \frac{\partial u}{\partial y}
\end{gathered}
$$

where the components of extra stress appearing in the above equations satisfy three equations that can be obtained directly from (4a).

The relevant boundary conditions are [8]

$$
u=0 \text {, }
$$

$$
\begin{array}{r}
{\left[-\tau \frac{\partial^{3}}{\partial x^{3}}+m \frac{\partial^{3}}{\partial x \partial t^{2}}+d \frac{\partial^{3}}{\partial x \partial t}\right] \eta} \\
=\frac{\partial S_{x x}}{\partial x}+\frac{\partial S_{x y}}{\partial y}-\rho \frac{d u}{d t}-\sigma B_{0}^{2} u \\
\text { at } y= \pm \eta
\end{array}
$$

where $\tau$ is the tension in the wall, $m$ is the mass per unit area, $d$ is the coefficient of viscous damping, and $\sigma$ is the electrical conductivity of fluid. Defining the stream function $\psi$ such that $u=\partial \psi / \partial y, v=-\partial \psi / \partial x$, and the following dimensionless variables:

$$
\begin{gathered}
\psi^{*}=\frac{\psi}{c d_{1}}, \quad x^{*}=\frac{x}{\lambda}, \quad y^{*}=\frac{y}{d_{1}}, \\
t^{*}=\frac{c t}{\lambda}, \quad \eta^{*}=\frac{\eta}{d_{1}}, \quad p^{*}=\frac{d_{1}^{2} p}{c \lambda \mu}, \\
S_{i j}^{*}=\frac{d_{1} S_{i j}}{c \mu}, \quad \lambda_{1}^{*}=\lambda_{1} \frac{c}{d_{1}}, \quad \lambda_{2}^{*}=\lambda_{2} \frac{c}{d_{1}},
\end{gathered}
$$

we find that the continuity equation (5) is identically satisfied and (8)-(9), after dropping the primes, reduce to

$$
\begin{aligned}
& R \delta\left(\frac{\partial}{\partial t}+\frac{\partial \psi}{\partial y} \frac{\partial}{\partial x}-\frac{\partial \psi}{\partial x} \frac{\partial}{\partial y}\right)\left(\delta^{2} \frac{\partial^{2} \psi}{\partial x^{2}}+\frac{\partial^{2} \psi}{\partial y^{2}}\right) \\
& =\left(\frac{\partial^{2}}{\partial y^{2}}-\delta^{2} \frac{\partial^{2}}{\partial x^{2}}\right) S_{x y}-M^{2} \frac{\partial^{2} \psi}{\partial y^{2}} \\
& +\delta \frac{\partial^{2}}{\partial x \partial y}\left(S_{x x}-S_{y y}\right),
\end{aligned}
$$




$$
\begin{gathered}
{\left[E_{1} \frac{\partial^{3}}{\partial x^{3}}+E_{2} \frac{\partial^{3}}{\partial x \partial t^{2}}+E_{3} \frac{\partial^{3}}{\partial x \partial t}\right] \eta} \\
=\delta \frac{\partial S_{x x}}{\partial x}-M^{2} \frac{\partial \psi}{\partial y}+\frac{\partial S_{x y}}{\partial y} \\
-R \delta\left(\frac{\partial}{\partial t}+\frac{\partial \psi}{\partial y} \frac{\partial}{\partial x}-\frac{\partial \psi}{\partial x} \frac{\partial \psi}{\partial y}\right) \frac{\partial}{\partial y}, \\
\psi_{y}=0 \quad \text { at } y= \pm \eta .
\end{gathered}
$$

In the above equations $\eta= \pm(1+\varepsilon \sin 2 \pi(x-t)), \varepsilon=a / d_{1}$ is the dimensionless amplitude, $\delta=d_{1} / \lambda$ denotes the characteristic ratio of transversal and axial scales of peristaltic motion, $R=$ $c d_{1} / \nu$ is the Reynolds number, $M=\sqrt{\sigma / \mu} B_{0} d_{1}$ denotes the Hartman number, $E_{1}=-\tau d_{1}^{3} / \lambda^{3} \mu c, E_{2}=\operatorname{mcd}_{1}^{3} / \lambda^{3} \mu, E_{3}=$ $d d_{1}^{3} / \lambda^{3} \mu$ are the nondimensional elasticity parameters, and $\beta^{*}=\beta c^{2} / d_{1}^{2}$ is the dimensionless Burger's fluid parameter.

\section{Solution of the Problem}

Due to nonlinearity of (11) it seems impossible to get a closed form solution. Therefore, some plausible assumptions must be made to put these equations in tractable form. We would like to mention here that without such assumptions one cannot obtain solution of these equations even for Newtonian fluid. Following [1], we assume that the $\delta$ is small and proceed to write

$$
\begin{gathered}
\psi=\sum_{k=0}^{\infty} \delta^{k} \psi_{k}, \\
S_{i j}=\sum_{k=0}^{\infty} \delta^{k} S_{i j k}, \quad i, j=1,2 .
\end{gathered}
$$

Substituting expressions (13) into (11) and (12) and collecting the terms of equal powers of $\delta$, we get the following systems.

\subsection{Zero-Order System}

$$
\begin{gathered}
\frac{\partial^{2} S_{0 x y}}{\partial y^{2}}-M^{2} \frac{\partial^{2} \psi_{0}}{\partial y^{2}}=0 \\
S_{0 y y}=0 \\
S_{0 x x}-2 \lambda_{1} \frac{\partial^{2} \psi_{0}}{\partial y^{2}} S_{0 x y}-2 \lambda_{2}\left(\frac{\partial^{2} \psi_{0}}{\partial y^{2}}\right)^{2}, \\
S_{0 x y}-\lambda_{1} \frac{\partial^{2} \psi_{0}}{\partial y^{2}} S_{0 y y}=\frac{\partial^{2} \psi_{0}}{\partial y^{2}}, \\
\frac{\partial^{3}}{\partial x^{3}}+E_{2} \frac{\partial^{2}}{\partial x t^{2}}+E_{3} \frac{\partial x \partial t}{\partial \psi_{0}}=0, \quad \text { at } y= \pm \eta .
\end{gathered}
$$

\subsection{First-Order System}

$$
\begin{aligned}
& R\left(\frac{\partial}{\partial t}+\frac{\partial \psi_{0}}{\partial y} \frac{\partial}{\partial x}-\frac{\partial \psi_{0}}{\partial x} \frac{\partial}{\partial y}\right) \frac{\partial^{2} \psi_{0}}{\partial y^{2}}+M^{2} \frac{\partial^{2} \psi_{1}}{\partial y^{2}} \\
& =\frac{\partial^{2} S_{1 x y}}{\partial y^{2}}+\frac{\partial^{2}}{\partial x \partial y}\left(S_{0 x x}-S_{0 y y}\right), \\
& S_{1 x x}+\lambda_{1}\left[\left(\frac{\partial}{\partial t}+\frac{\partial \psi_{0}}{\partial y} \frac{\partial}{\partial x}-\frac{\partial \psi_{0}}{\partial x} \frac{\partial}{\partial y}\right) S_{0 x x}\right. \\
& -2 \frac{\partial^{2} \psi_{0}}{\partial x \partial y} S_{0 x x} \\
& \left.-2 \frac{\partial^{2} \psi_{1}}{\partial y^{2}} S_{0 x y}-2 \frac{\partial^{2} \psi_{0}}{\partial y^{2}} S_{1 x y}\right] \\
& +\beta\left[\left\{4 \frac{\partial^{2} \psi_{0}}{\partial x \partial y} \frac{\partial^{2} \psi_{0}}{\partial y^{2}}\right.\right. \\
& \left.-2\left(\frac{\partial \psi_{0}}{\partial y} \frac{\partial^{3} \psi_{0}}{\partial x \partial y^{2}}-\frac{\partial \psi_{0}}{\partial x} \frac{\partial^{3} \psi_{0}}{\partial y^{3}}\right)\right\} S_{0 x y} \\
& -2\left(\frac{\partial^{2} \psi_{0}}{\partial x \partial y}\right)^{2} S_{1 y y} \\
& \times\left\{-4 \frac{\partial^{2} \psi_{0}}{\partial y^{2}}\left(\frac{\partial \psi_{0}}{\partial y} \frac{\partial}{\partial x}-\frac{\partial \psi_{0}}{\partial x} \frac{\partial}{\partial y}\right)\right\} S_{0 x y} \\
& \left.-2\left(\frac{\partial^{2} \psi_{0}}{\partial y^{2}} \frac{\partial^{2} \psi_{1}}{\partial y^{2}}\right)^{2} S_{0 y y}\right] \\
& =2 \frac{\partial^{2} \psi_{0}}{\partial x \partial y}-4 \lambda_{2} \frac{\partial^{2} \psi_{0}}{\partial y^{2}} \frac{\partial^{2} \psi_{1}}{\partial y^{2}} \\
& S_{1 y y}+\lambda_{1}\left[\left(\frac{\partial}{\partial t}+\frac{\partial \psi_{0}}{\partial y} \frac{\partial}{\partial x}-\frac{\partial \psi_{0}}{\partial x} \frac{\partial}{\partial y}\right) S_{0 y y}+2 \frac{\partial^{2} \psi_{0}}{\partial x \partial y} S_{0 y y}\right] \\
& =-2 \frac{\partial^{2} \psi_{0}}{\partial x \partial y} \\
& S_{1 x y}+\lambda_{1}\left[\left(\frac{\partial}{\partial t}+\frac{\partial \psi_{0}}{\partial y} \frac{\partial}{\partial x}-\frac{\partial \psi_{0}}{\partial x} \frac{\partial}{\partial y}\right) S_{0 x y}\right. \\
& \left.-\frac{\partial^{2} \psi_{0}}{\partial y^{2}} S_{1 x y}-\frac{\partial^{2} \psi_{1}}{\partial y^{2}} S_{0 y y}\right] \\
& =\frac{\partial^{2} \psi_{1}}{\partial y^{2}}+\lambda_{2}\left[\left(\frac{\partial}{\partial t}+\frac{\partial \psi_{0}}{\partial y} \frac{\partial}{\partial x}-\frac{\partial \psi_{0}}{\partial x} \frac{\partial}{\partial y}\right) \frac{\partial^{2} \psi_{0}}{\partial y^{2}}\right. \\
& \left.+2 \frac{\partial^{2} \psi_{0}}{\partial x \partial y} \frac{\partial^{2} \psi_{0}}{\partial y^{2}}\right]
\end{aligned}
$$




$$
\begin{gathered}
\frac{\partial S_{0 x x}}{\partial x}+\frac{\partial S_{1 x y}}{\partial y} \\
-R\left(\frac{\partial}{\partial t}+\frac{\partial \psi_{0}}{\partial y} \frac{\partial}{\partial x}-\frac{\partial \psi_{0}}{\partial x} \frac{\partial}{\partial y}\right) \frac{\partial \psi_{0}}{\partial y}-M^{2} \frac{\partial \psi_{1}}{\partial y}=0, \\
\psi_{1 y}=0, \quad \text { at } y= \pm \eta .
\end{gathered}
$$

\subsection{Second-Order System}

$$
\begin{aligned}
& R\left[\left(\frac{\partial}{\partial t}+\frac{\partial \psi_{0}}{\partial y} \frac{\partial}{\partial x}-\frac{\partial \psi_{0}}{\partial x} \frac{\partial}{\partial y}\right) \frac{\partial^{2} \psi_{1}}{\partial y^{2}}\right. \\
& \left.+\left(\frac{\partial \psi_{1}}{\partial y} \frac{\partial}{\partial x}-\frac{\partial \psi_{1}}{\partial x} \frac{\partial}{\partial y}\right) \frac{\partial^{2} \psi_{0}}{\partial y^{2}}\right] \\
& =\frac{\partial^{2} S_{2 x y}}{\partial y^{2}}-\frac{\partial^{2} S_{0 x y}}{\partial x^{2}}-M^{2} \frac{\partial^{2} \psi_{2}}{\partial y^{2}} \\
& +\frac{\partial^{2}}{\partial x \partial y}\left(S_{1 x x}-S_{1 y y}\right), \\
& S_{2 x y}+\lambda_{1}\left[\left(\frac{\partial \psi_{1}}{\partial y} \frac{\partial}{\partial x}-\frac{\partial \psi_{1}}{\partial x} \frac{\partial}{\partial y}\right) S_{0 x y}\right. \\
& +\left(\frac{\partial}{\partial t}+\frac{\partial \psi_{0}}{\partial y} \frac{\partial}{\partial x}-\frac{\partial \psi_{0}}{\partial x} \frac{\partial}{\partial y}\right) S_{1 x y} \\
& +\frac{\partial^{2} \psi_{0}}{\partial x^{2}} S_{0 x x} \\
& \left.-\frac{\partial^{2} \psi_{0}}{\partial y^{2}} S_{2 y y}-\frac{\partial^{2} \psi_{1}}{\partial y^{2}} S_{1 y y}-\frac{\partial^{2} \psi_{2}}{\partial y^{2}} S_{0 y y}\right] \\
& +\beta\left\{\frac{\partial^{2}}{\partial t^{2}}+\frac{\partial}{\partial t}\left(\frac{\partial \psi_{0}}{\partial y} \frac{\partial}{\partial x}-\frac{\partial \psi_{0}}{\partial x} \frac{\partial}{\partial y}\right)\right. \\
& +\left(\frac{\partial \psi_{0}}{\partial y} \frac{\partial}{\partial x}-\frac{\partial \psi_{0}}{\partial x} \frac{\partial}{\partial y}\right) \\
& \times \frac{\partial}{\partial t}\left(\frac{\partial \psi_{0}}{\partial y} \frac{\partial}{\partial x}-\frac{\partial \psi_{0}}{\partial x} \frac{\partial}{\partial y}\right)^{2} \\
& \left.-4 \frac{\partial^{2} \psi_{0}}{\partial y^{2}} \frac{\partial \psi_{0}}{\partial x^{2}}\right\} S_{0 x y} \\
& +\left\{-2 \frac{\partial^{2} \psi_{0}}{\partial x \partial y} \frac{\partial}{\partial t}-2\left(\frac{\partial^{2} \psi_{0}}{\partial x \partial y}\right)^{2}\right. \\
& -2 \frac{\partial^{2} \psi_{0}}{\partial x \partial y}\left(\frac{\partial \psi_{0}}{\partial y} \frac{\partial}{\partial x}-\frac{\partial \psi_{0}}{\partial x} \frac{\partial}{\partial y}\right) \\
& \left.-\left(\frac{\partial \psi_{0}}{\partial y} \frac{\partial^{3} \psi_{0}}{\partial x^{2} \partial y}-\frac{\partial \psi_{0}}{\partial x} \frac{\partial^{3} \psi_{0}}{\partial x \partial y^{2}}\right)\right\} S_{0 y y}
\end{aligned}
$$

$$
\begin{aligned}
& =\left(\frac{\partial^{2} \psi_{2}}{\partial y^{2}}-\frac{\partial^{2} \psi_{0}}{\partial x^{2}}\right) \\
& +\lambda_{2}\left[\left(\frac{\partial}{\partial t}+\frac{\partial \psi_{0}}{\partial y} \frac{\partial}{\partial x}-\frac{\partial \psi_{0}}{\partial x} \frac{\partial}{\partial y}\right) \frac{\partial^{2} \psi_{1}}{\partial y^{2}}\right. \\
& +\left(\frac{\partial \psi_{1}}{\partial y} \frac{\partial}{\partial x}-\frac{\partial \psi_{1}}{\partial x} \frac{\partial}{\partial y}\right) \frac{\partial^{2} \psi_{0}}{\partial y^{2}} \\
& \left.+2 \frac{\partial^{2} \psi_{1}}{\partial x \partial y} \frac{\partial^{2} \psi_{0}}{\partial y^{2}}+2 \frac{\partial^{2} \psi_{0}}{\partial x \partial y} \frac{\partial^{2} \psi_{1}}{\partial y^{2}}\right], \\
& S_{2 y y}+\lambda_{1}\left[\left(\frac{\partial}{\partial t}+\frac{\partial \psi_{0}}{\partial y} \frac{\partial}{\partial x}-\frac{\partial \psi_{0}}{\partial x} \frac{\partial}{\partial y}\right) S_{i y y}\right. \\
& \left.-2 \frac{\partial^{2} \psi_{0}}{\partial x \partial y} S_{1 y y}-2 \frac{\partial^{2} \psi_{0}}{\partial x^{2}} S_{0 x y}\right] \\
& +\beta\left[\left\{\frac{\partial}{\partial t^{2}}+\frac{\partial}{\partial t}\left(\frac{\partial \psi_{0}}{\partial y} \frac{\partial}{\partial x}-\frac{\partial \psi_{0}}{\partial x} \frac{\partial}{\partial y}\right)\right.\right. \\
& +\left(\frac{\partial \psi_{0}}{\partial y} \frac{\partial}{\partial x}-\frac{\partial \psi_{0}}{\partial x} \frac{\partial}{\partial y}\right)^{2} \\
& -2 \frac{\partial^{3} \psi_{0}}{\partial x \partial y \partial t}+4 \frac{\partial^{2} \psi_{0}}{\partial x \partial y} \frac{\partial}{\partial t} \frac{\partial}{\partial t} \\
& +\left(\frac{\partial \psi_{0}}{\partial y} \frac{\partial}{\partial x}-\frac{\partial \psi_{0}}{\partial x} \frac{\partial}{\partial y}\right) \\
& +\left(\frac{\partial \psi_{0}}{\partial y} \frac{\partial}{\partial x}-\frac{\partial \psi_{0}}{\partial x} \frac{\partial}{\partial y}\right) \frac{\partial^{2} \psi_{0}}{\partial x \partial y}+4\left(\frac{\partial^{2} \psi_{0}}{\partial x \partial y}\right)^{2} \\
& +\frac{\partial^{2} \psi_{0}}{\partial x \partial y}\left(\frac{\partial \psi_{0}}{\partial y} \frac{\partial}{\partial x}-\frac{\partial \psi_{0}}{\partial x} \frac{\partial}{\partial y}\right) \\
& \left.\left.+2 \frac{\partial^{2} \psi_{0}}{\partial y^{2}} \frac{\partial^{2} \psi_{0}}{\partial x^{2}}\right\} S_{0 y y}\right] \\
& =-2 \frac{\partial^{2} \psi_{1}}{\partial x \partial y} \\
& -2 \lambda_{2}\left[\left(\frac{\partial}{\partial t}+\frac{\partial \psi_{0}}{\partial y} \frac{\partial}{\partial x}-\frac{\partial \psi_{0}}{\partial x} \frac{\partial}{\partial y}\right) \frac{\partial^{2} \psi_{0}}{\partial x \partial y}\right. \\
& \left.-2\left(\frac{\partial^{2} \psi_{0}}{\partial x \partial y}\right)^{2}+\frac{\partial^{2} \psi_{0}}{\partial x^{2}} \frac{\partial^{2} \psi_{0}}{\partial y^{2}}\right] \\
& \frac{\partial S_{1 x x}}{\partial x}+\frac{\partial S_{2 x y}}{\partial y}-M^{2} \frac{\partial \psi_{2}}{\partial y} \\
& -R\left[\left(\frac{\partial}{\partial t}+\frac{\partial \psi_{0}}{\partial y} \frac{\partial}{\partial x}-\frac{\partial \psi_{0}}{\partial x} \frac{\partial}{\partial y}\right) \frac{\partial \psi_{1}}{\partial y}\right. \\
& \left.+\left(\frac{\partial \psi_{1}}{\partial y} \frac{\partial}{\partial x}-\frac{\partial \psi_{1}}{\partial x} \frac{\partial}{\partial y}\right) \frac{\partial \psi_{0}}{\partial y}\right]=0, \\
& \frac{\partial \psi_{2}}{\partial y}=0, \quad \text { at } y= \pm \eta
\end{aligned}
$$




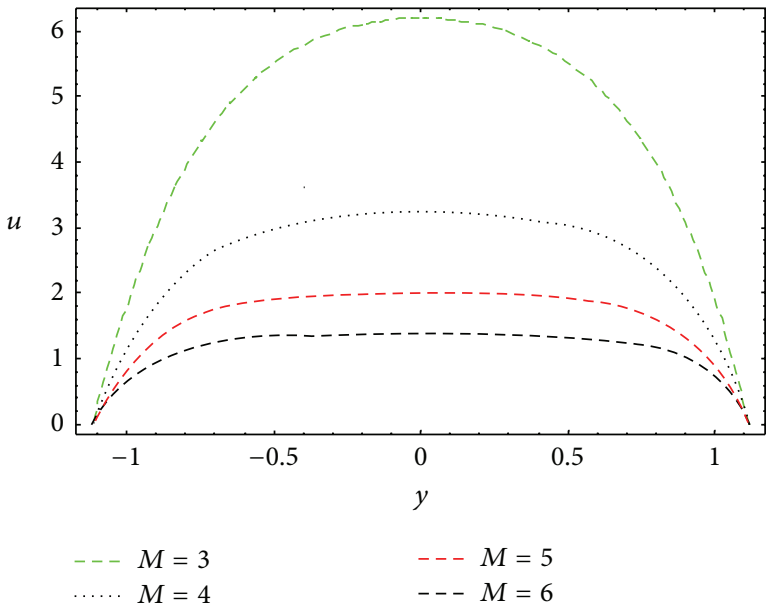

(a)

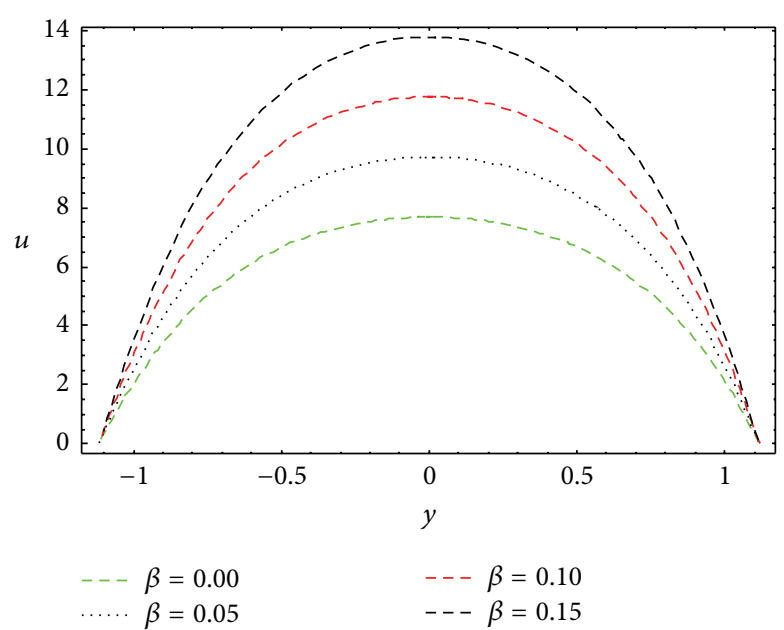

(b)

Figure 1: Variation on $u$ when $E_{1}=1, E_{2}=0.2, E_{3}=0.5, \varepsilon=0.2, \delta=0.01, t=0.2, R=0.01, \lambda_{1}=0.1, \lambda_{2}=0.05$, and $x=0.3$ plotted for (a) for different values of $M$ and (b) different Burger's fluid parameter $\beta$.

The solution of these systems yields

$$
u=u_{0}+\delta u_{1}+\delta^{2} u_{2}
$$

where

$$
\begin{gathered}
u_{0}=L(\operatorname{sech} M \eta \cosh M y-1), \\
u_{1}=A_{2} y+A_{4} \sinh M y+L_{2} y \cosh M y+L_{3} y^{2} \sinh M y
\end{gathered}
$$

and value of $u_{2}$ is given in the appendix.

The value of constants involved in (18) is given in the appendix.

\section{Results and Discussion}

The purpose of this section is to provide the graphical illustration of the solution obtained in previous section. Emphasis has been given to examine the influence of emerging parameters such as $\delta, R, M$, and the rheological parameter of the Burger's fluid $\beta$ on the axial velocity and the stream function.

In fact the novelty of the present analysis is based on the effects of Burger's fluid parameter $\beta$ on various flow characteristics. This is because of the fact that the results in the absence of $\beta$ are available already in the literature [13] for comparison. Particularly the velocity $u$ is plotted for different values of the involved parameter including the Burger's fluid parameter $\beta$. Figure 1 interprets the effects of Hartman number $M$ and $\beta$ on $u$. It is noted from this figure that $u$ is an increasing function of $\beta$, while it decreases with $M$. The decrease of $u$ with $M$ is a result of the fact that magnetic force acts in the transverse direction and hence resists the flow due to peristalsis.

The effects of $R$ and $\delta$ on $u$ can be seen through Figure 2 . This figure shows that $u$ increases by increasing $R$ and $\delta$. The Reynolds number represents the relative magnitude of inertial to the viscous force and with the increase of Reynolds number, the viscous forces decrease resulting in increases in velocity. Similarly with an increase in $\delta$ the wavelength of wave decreases which causes the increase in the velocity. It is worth mentioning that these results are in accordance with those obtained in [11] for a Maxwell fluid. A maximum in the velocity at center of the channel and an increase of velocity with $R$ and $\delta$ are also observed from Figure 2 .

Figure 3 is prepared to see the effects of complaint wall parameters $E_{1}, E_{2}$, and $E_{3}$ on longitudinal distribution of velocity $u$. This figure reveals that the velocity increases when both wall parameters $E_{1}$ and $E_{2}$ increase because of the increase in flexibility and elasticity of the wall. However, velocity decreases with $E_{3}$ due to the increase in rigidity of the wall. Note that we recover the results reported in [11] for $\lambda_{2}=\beta=0$.

Figure 4 illustrates the effects of $\lambda_{1}$ and $\lambda_{2}$ on $u$. It is observed that when we increase the relaxation parameter the velocity $u$ gets increased due to the increment in $\lambda_{1}$. The effects of retardation parameter $\lambda_{2}$ on the velocity are quite opposite to that of $\lambda_{1}$.

The purpose of Figures 5, 6, 7, and 8 is to capture the variation in size and circulation of the trapped bolus, in particular, the effects of $E_{1}, E_{2}, \beta$, and $E_{3}$. It is observed from these figures that the size of bolus decreases by increasing $E_{1}$ and $E_{2}$ while it increases with $E_{3}$ and $\beta$.

\section{Conclusions}

Peristaltic motion of a (MHD) non-Newtonian fluid with Burger's constitutive equation in channel with complaint walls is taken into account. The results for velocity and stream function are obtained and compared with the literature. It is observed that Reynolds number and $\delta$ cause an increase in the velocity while a decrease in velocity is noted by increasing Hartman number. In previous mathematical model [13], Burger's parameter is absent and therefore such models 


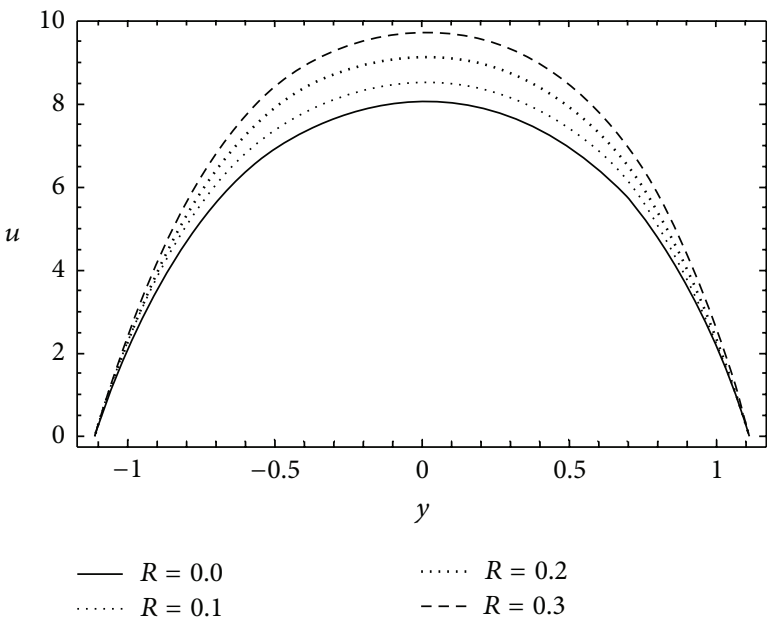

(a)

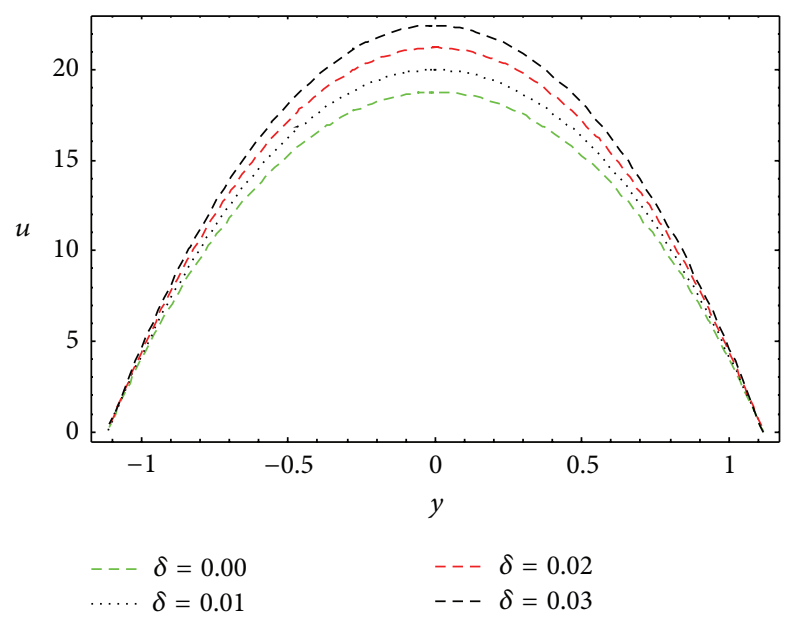

(b)

FIGURE 2: Variation on $u$ when $E_{1}=1, E_{2}=0.2, E_{3}=0.5, \varepsilon=0.2, \beta=0.05, t=0.2, M=2.5, \lambda_{1}=0.1, \lambda_{2}=0.0$, and $x=0.3$ plotted for (a) for different values of $R$ and (b) different values of $\delta$.

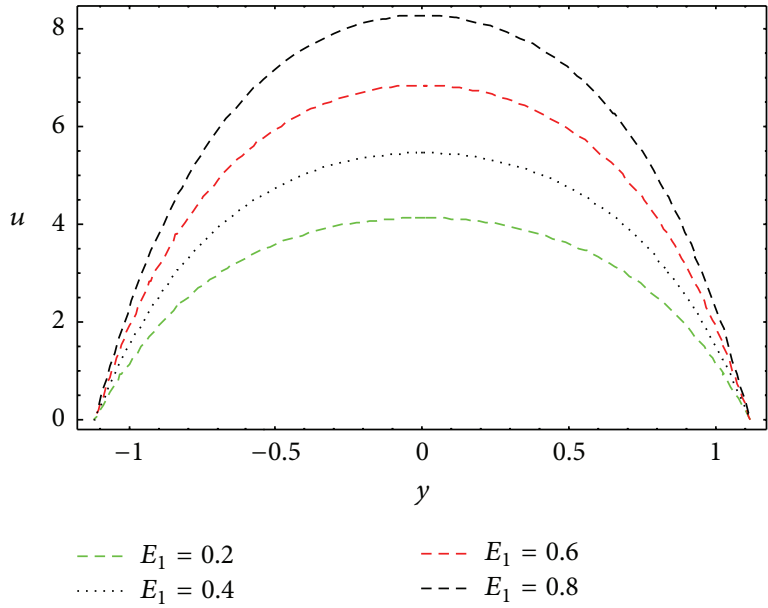

(a)

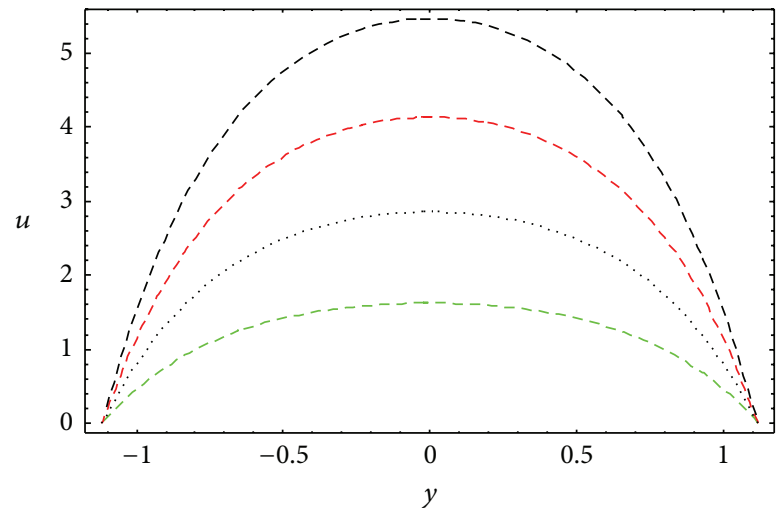

$\begin{array}{ll}---E_{2}=0.2 \\ \cdots \cdots & E_{2}=0.4\end{array}$

$--E_{2}=0.6$

$--E_{2}=0.8$

(b)

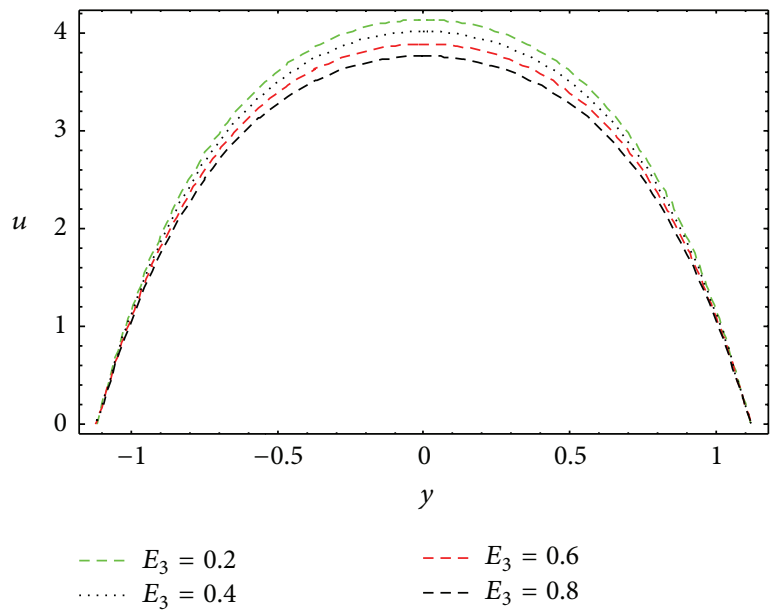

(c)

Figure 3: Variation on $u$ when $R=0.01, \delta=0.01, \varepsilon=0.2, \beta=0.01, t=0.2, M=2.6, \lambda_{1}=0.1, \lambda_{2}=0.0$, and $x=0.3$ plotted for (a) for different values of $E_{1}$, (b) for different values of $E_{2}$, and (c) for different values of $E_{3}$. 


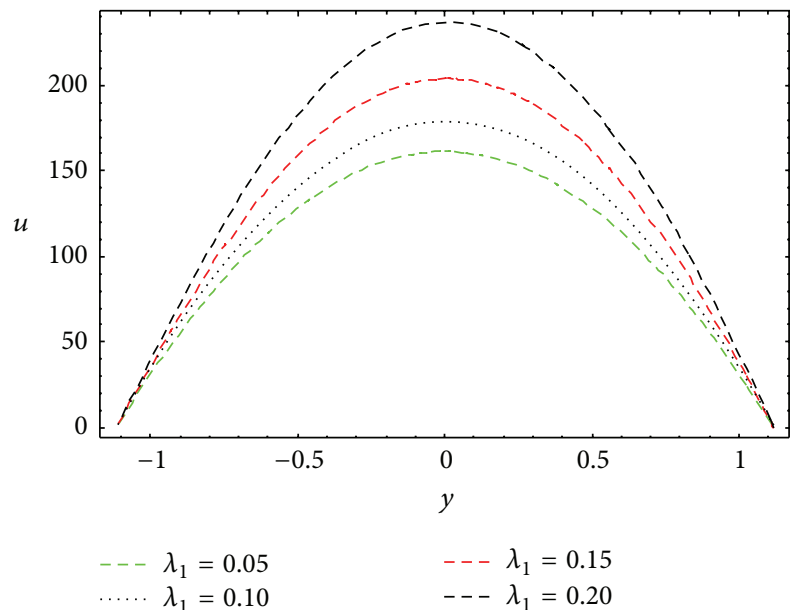

(a)

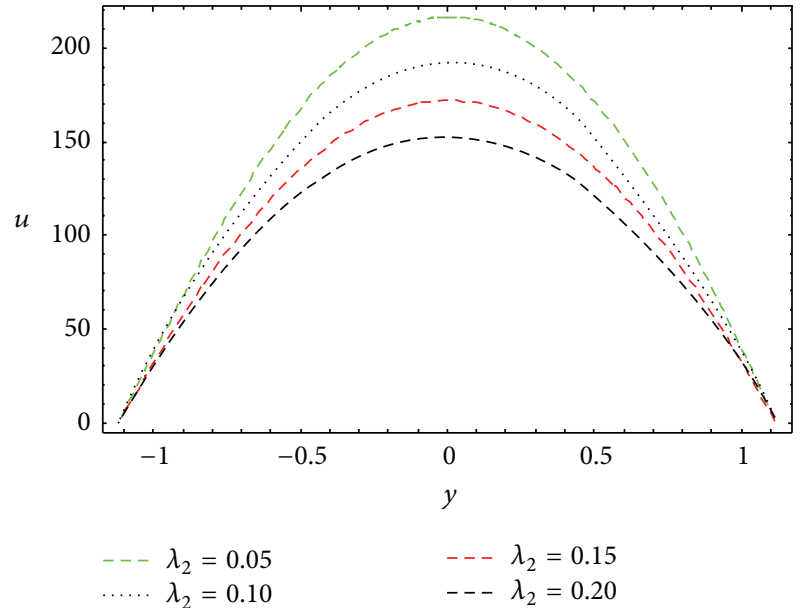

(b)

Figure 4: Variation on $u$ when $E_{1}=1, E_{2}=0.2, E_{3}=0.5, \varepsilon=0.2, \beta=0.05, t=0.2, M=1, \delta=0.01, R=0.01$, and $x=0.3$ plotted for (a) for different values of $\lambda_{1}$ and (b) for different values of $\lambda_{2}$.

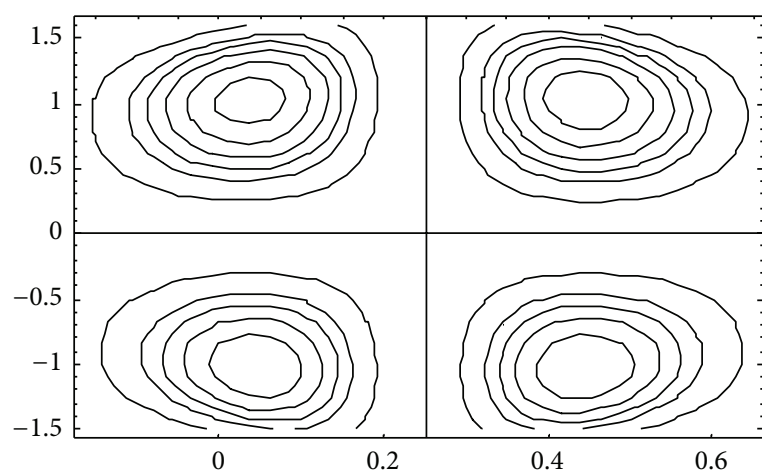

(a)

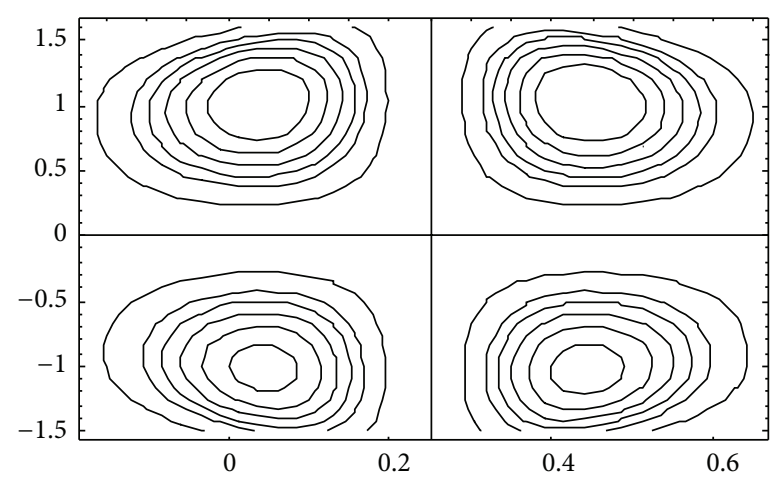

(b)

Figure 5: Streamlines when $E_{1}=0.2, E_{2}=0.1, E_{3}=0.1, \varepsilon=0.1, \delta=0.01, t=0.0, R=0.01, M=1, \lambda_{1}=0.1$, and $\lambda_{2}=0.05$. (a) $\beta=0.01$ and (b) $\beta=0.05$.

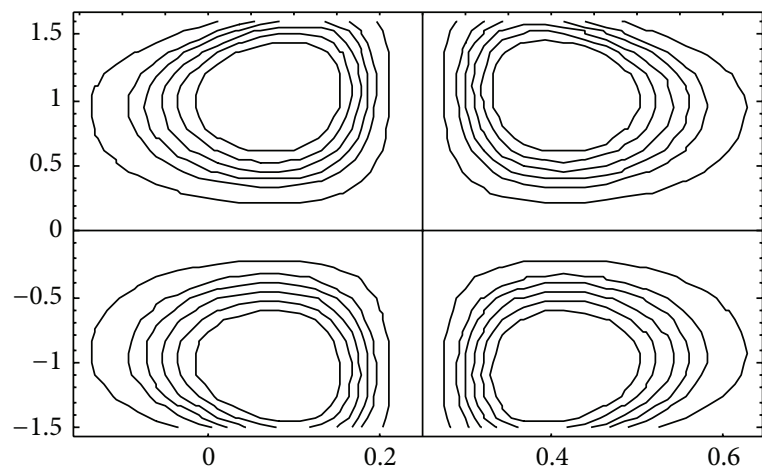

(a)

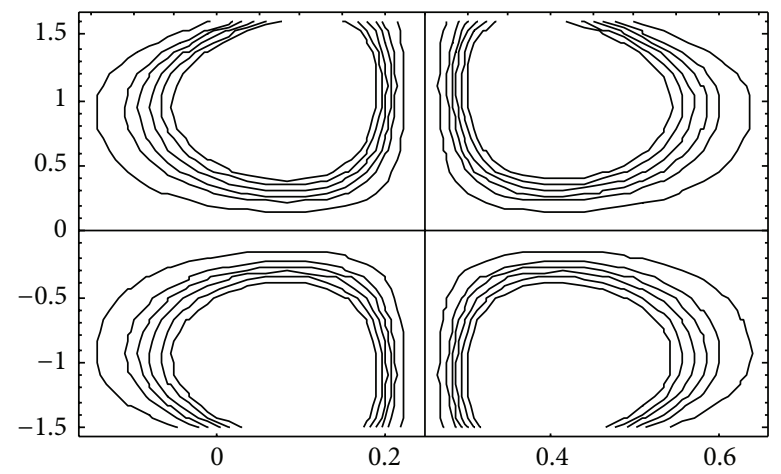

(b)

Figure 6: Streamlines when $\beta=0.05, E_{2}=0.1, E_{3}=0.1, \varepsilon=0.1, \delta=0.01, t=0.0, R=0.01, M=1, \lambda_{1}=0.1$, and $\lambda_{2}=0.05$. (a) $E_{1}=0.1$ and (b) $E_{1}=0.3$. 


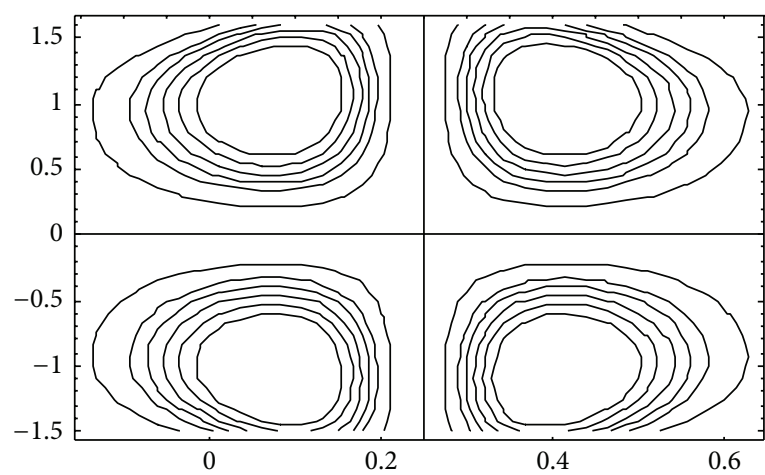

(a)

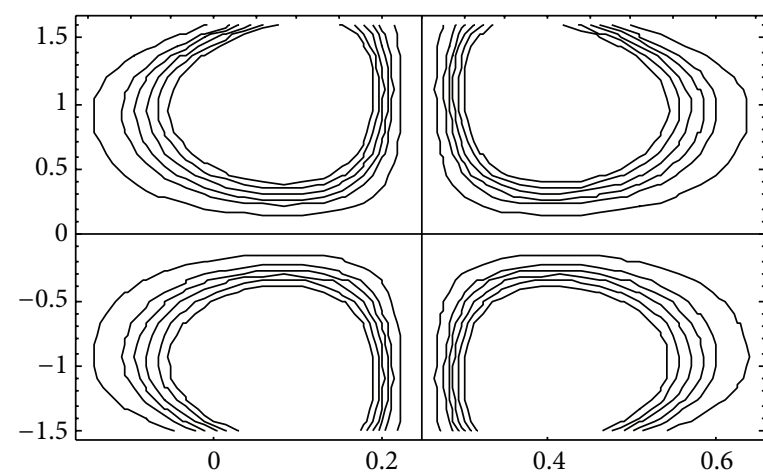

(b)

Figure 7: Streamlines when $\beta=0.05, E_{1}=0.1, E_{3}=0.1, \varepsilon=0.1, \delta=0.01, t=0.0, R=0.01, M=1, \lambda_{1}=0.1$, and $\lambda_{2}=0.05$. (a) $E_{2}=0.1$ and (b) $E_{2}=0.3$.

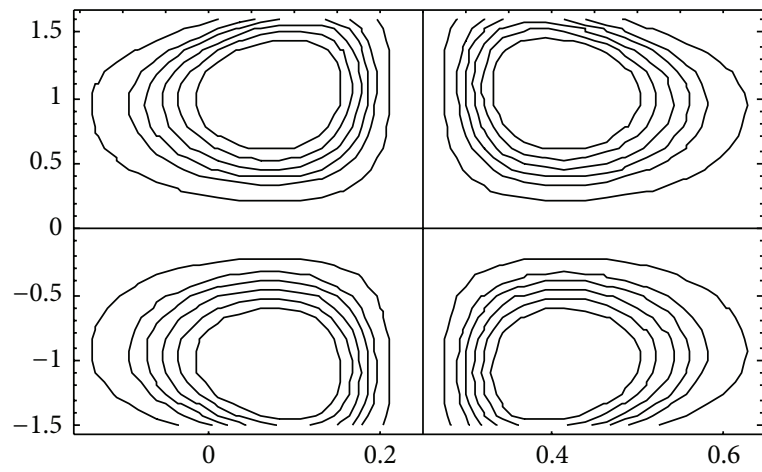

(a)

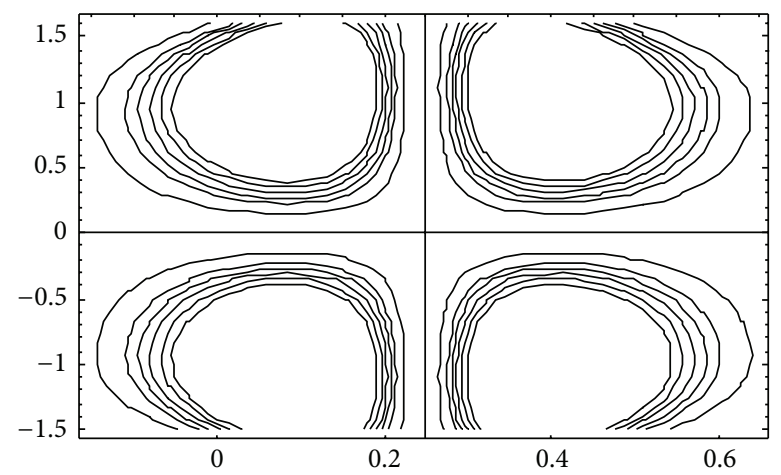

(b)

Figure 8: Streamlines when $\beta=0.05, E_{1}=0.1, E_{2}=0.1, \varepsilon=0.1, \delta=0.01, t=0.0, R=0.01, M=1, \lambda_{1}=0.1$, and $\lambda_{2}=0.05$. (a) $E_{3}=0.1$ and (b) $E_{3}=0.5$.

cannot accurately describe the peristaltic motion of food stuff like cheese and so forth. The physical impact of this parameter is quite interesting. Our mathematical model predicts an increase in velocity by increasing Burger's fluid parameter. The model further reveals that the size and circulation of the trapped bolus increase with Burger's fluid parameter.

\section{Appendix}

Here we provide the value of $u_{2}$ along with various constants appearing in it and in the expressions of $u_{0}$ and $u_{1}$, as follows:

$$
\begin{aligned}
u_{2}=\frac{1}{4 M}( & 2 a_{49} \\
& \\
& +M\left(4 c_{4} M+2 a_{53} y\right. \\
& \left.\left.\quad+a_{52} y^{2}+4 a_{48} y^{2}+4 a_{38} y^{4}\right)\right) \cosh M y
\end{aligned}
$$

$$
\begin{aligned}
& -\frac{1}{4 M^{4}}\left(a_{27 x}+24 a_{54}\right. \\
& +M^{2}\left(2 a_{51}+4 a_{55}+4 c_{1}+\left(a_{27 x}+12 a_{54}\right) y^{2}\right. \\
& \left.\left.+M\left(2 a_{49} \cosh M y-a_{47}\right)\right)\right) \cosh ^{2} M y \\
& +\frac{1}{108 M^{4}} \\
& \times\left(\left(3 M\left(a_{27 x} y-3 a_{36} M-12 M^{2} a_{51} y\right)\right) \cosh M y\right. \\
& +\left(a_{27 x}+3 M^{2}\left(2 a_{51}+12 M a_{47}-3 a_{27 x} y^{2}\right)\right) \\
& \quad \times \sinh M y) \sinh 3 M y \\
& +\frac{1}{2 M^{3}}\left(a_{27 x} y+M\left(a_{36}+4 M a_{51} y\right)\right) \cosh M y \sinh M y \\
& +\frac{1}{8 M}\left(3 a_{46} \sinh M y-3 a_{50} \cosh M y\right) \sinh 4 M y
\end{aligned}
$$




$$
\begin{aligned}
& +\frac{1}{4 M^{4}}\left(24 a_{54}-a_{27 x}\right. \\
& +M^{2}\left(4 \left(a_{55}+c_{1}\right.\right. \\
& \left.+M\left(a_{47}+a_{49} \cosh M y\right)+3 a_{54} y^{2}\right) \\
& \left.\left.-a_{27 x} y^{2}-2 a_{51}\right)\right) \sinh ^{2} M y \\
& +\frac{1}{16 M^{5}} \\
& \times\left(-3 a_{38}+2 M^{2}\left(a_{48}-M a_{52}+2 M^{2}\left(3 a_{46}-2 a_{49}\right)\right.\right. \\
& \left.\left.-\left(3 a_{38}+2 a_{48} M^{2}\right) y^{2}\right)\right) \cosh M y \cosh 2 M y \\
& +\frac{1}{8 M^{4}}\left(2 M^{2}\left(6 M a_{50}-M a_{53}-a_{48} y-M a_{52} y-a_{38} y^{3}\right)\right. \\
& \left.+3 a_{38} y\right) \cosh M y \sinh 2 M y \\
& +\frac{1}{8 M^{4}} \\
& \times\left(2 M^{3}\left(3 a_{50}+a_{53}+a_{52} y\right)\right. \\
& \left.+2 M^{2}\left(a_{48} y+a_{38} y^{3}\right)-3 a_{38} y\right) \cosh 2 M y \sinh M y \\
& +\left(\frac{1}{108 M^{4}}\right. \\
& \times\left(-a_{27 x}-6 a_{51} M^{2}-36 a_{47} M^{3}+9 a_{27 x} M^{2} y^{2}\right) \\
& \left.\times \cosh 3 M y-\frac{3 a_{46}}{8 M} \cosh 4 M y\right) \cosh M y \\
& +\frac{1}{6 M^{2}}\left(3\left(a_{53}+2 M^{2} c_{3}+a_{49} M^{2} y+a_{52} M y+a_{38} y^{3}\right)\right. \\
& \left.+a_{48} y^{2} M^{2}\right) \sinh M y \\
& +\frac{1}{16 M^{5}}\left(3 a_{38}\right. \\
& +2 M^{2}\left(a_{52}-a_{48}+2 M^{2}\left(3 a_{46}+a_{49}+a_{48} y^{2}\right)\right. \\
& \left.\left.+3 a_{38} y^{2}\right)\right) \sinh M y \sinh 2 M y \\
& +\left(\frac{1}{36 M^{3}}\left(3 a_{36} M-a_{27 x} y+12 M^{2} a_{51} y\right)\right. \\
& \left.\times \cosh 3 M y+\frac{3 a_{50}}{8 M} \cosh 4 M y\right) \sinh M y, \\
& A_{2}=\frac{1}{M^{2}}\left\{\left\langle2 L_{2} M^{2}+6 M L_{3}\right.\right. \\
& \left.-L_{1}(1+L)\left(\frac{\operatorname{Re}}{M}+M\left(\lambda_{1}-\lambda_{2}\right)\right)\right\rangle \cosh M \eta \\
& +\left\{4 M^{2} L_{3}\right. \\
& \left.+\frac{M L L_{t}}{\cosh M \eta}\left(\operatorname{Re}+M^{2}\left(\lambda_{1}-\lambda_{2}\right)\right)\right\} \eta \sinh M \eta+\operatorname{Re} L_{t} \\
& +\frac{\left(\operatorname{Re}+2 M^{2}\left(\lambda_{1}-\lambda_{2}\right) L L_{1}\right)}{M \cosh M \eta} \\
& \left.-M^{2} L L_{t}\left(\lambda_{1}-\lambda_{2}\right)\right\}
\end{aligned}
$$

$$
\begin{aligned}
& A_{4}=\frac{-1}{M \cosh M \eta} \\
& \times\left\{A_{2}+\left(M L_{2}+2 L_{3}\right) \eta \sinh M \eta\right. \\
& \left.+\left(L_{2}+M L_{3} \eta^{2}\right) \cosh M \eta\right\}, \\
& L_{1}=\frac{M L_{t}}{\cosh M \eta}-\frac{\eta_{t} M^{2} L \sinh M \eta}{\cosh ^{2} M \eta}, \\
& L_{2}=\frac{R+\left(\lambda_{1}-\lambda_{2}\right) M^{2}}{2 M^{2}}\left\{\frac{L_{1}(1+L)}{M}+\frac{5 L L_{t}}{2 \cosh M \eta}\right\} \text {, } \\
& L_{3}=\frac{\left(R+\left(\lambda_{1}-\lambda_{2}\right) M^{2}\right) L L_{t}}{4 M \cosh M \eta} \\
& a_{11}=-\frac{L_{1 t}}{M^{2}}-\left(\lambda_{1}-\lambda_{2}\right) \\
& \times\left(4 L_{t} L_{3}+2 L_{3 t}+2 L L_{3 t}\right. \\
& +4 L_{t} L_{2} M+2 L_{2 t} M+2 L L_{2 t} M \\
& +A_{4 t} M^{2}+A_{4 t} L M^{2}+2 A_{4} L_{t} M^{2} \\
& \left.-2 A_{2 t} L M \operatorname{sech} M \eta-A_{2} L_{1}\right) \\
& +\lambda_{1}\left(\lambda_{1}-\lambda_{2}\right) \\
& \times\left(\left(1+2 L+3(1+L) L_{1}+4 M L L_{t} \operatorname{sech} M \eta\right) L_{t}\right. \\
& \left.+4 M(1+L) L_{t t} \operatorname{sech} M \eta\right) \\
& -\beta\left(\left(1+L_{t}\right) L_{1}+\left(2+L+L_{1}\right) L L_{t}\right), \\
& a_{12}=-\left(\lambda_{1}-\lambda_{2}\right) \\
& \times\left(4 L_{1} L_{3}-2 M\left(2 L_{3 t}+L_{2 t} M\right) L \operatorname{sech} M \eta\right) \\
& \text { - } \beta\left(4 L L_{t t} M \operatorname{sech} M \eta-L_{1} L_{t}\right) M \operatorname{sech} M \eta \text {, } \\
& a_{13}=-\left(\lambda_{1}-\lambda_{2}\right) \\
& \times\left(\left(-4 L_{3}-L_{2}\right) L_{t} M^{2}\right. \\
& \left.+(1+L-L \operatorname{sech} M \eta) L_{3 t} M^{2}-2 L_{1} L_{3} M\right) \\
& +\lambda_{1}\left(\lambda_{1}-\lambda_{2}\right)\left(L L_{t}^{2} M^{3} \operatorname{sech} M \eta\right) \\
& \text { - } \beta\left(L L_{t}^{2} M^{3} \operatorname{sech} M \eta\right) \text {, } \\
& a_{14}=-\left(\lambda_{1}-\lambda_{2}\right) \\
& \times\left(\left(-2 L_{2}+\frac{2 L_{3}}{M}-2 A_{4} M\right) L_{1}\right. \\
& \left.-\left(2 L_{3 t}+4 L_{2 t} M+2 A_{4 t} M^{2}\right) L \operatorname{sech} M \eta\right) \\
& +\lambda_{1}\left(\lambda_{1}-\lambda_{2}\right) \\
& \times\left(-(1+L) \frac{L_{1}^{2}}{M}\right. \\
& -\left(1+2 L_{1}-L\right) 5 L L_{t} \operatorname{sech} M \eta \\
& \left.+4 L^{2} L_{t t} M \operatorname{sech}^{2} M \eta\right) \\
& -\beta\left((1+L) \frac{L_{1}^{2}}{M}-\left(2+L+L_{1}\right) L L_{t} \operatorname{sech} M \eta\right),
\end{aligned}
$$




$$
\begin{aligned}
& a_{15}=-\left(\lambda_{1}-\lambda_{2}\right) \\
& \times\left(-2\left(4 L_{3}+L_{2} M\right) L_{1}-4 L L_{3 t} M \operatorname{sech} M \eta\right) \\
& +\lambda_{1}\left(\lambda_{1}-\lambda_{2}\right) \\
& \times\left(\left(3 L_{1} L_{t}+L L_{t t} M \operatorname{sech} M \eta\right) L M \operatorname{sech} M \eta\right) \\
& \text { - } \beta\left(\left(L_{1} L_{t}+L L_{t t} M \operatorname{sech} M \eta\right) L M \operatorname{sech} M \eta\right) \text {, } \\
& a_{16}=-\left(\lambda_{1}-\lambda_{2}\right) \\
& \times\left(\left(2 L_{3}-L_{2} M-A_{4} M^{2}\right) L_{t} M\right. \\
& \left.+4(1+L) L_{3 t} M+(1+L) L_{2 t} M\right) \\
& +A_{2 t} L M^{2} \operatorname{sech} M \eta \\
& +\lambda_{1}\left(\lambda_{1}-\lambda_{2}\right) \\
& \times\left(-2(1+L) L_{1} L_{t} M\right. \\
& \left.-\left(L_{t}^{2}+L_{t t}(1+L)\right) L M^{2} \operatorname{sech} M \eta\right) \\
& -\beta\left(-2(1+L) L_{1} L_{t} M\right. \\
& \left.+\left(L_{t}^{2}-L L_{t t}\right) L M^{2} \operatorname{sech} M \eta\right), \\
& a_{17}=\lambda_{1}\left(\lambda_{1}-\lambda_{2}\right)\left(\frac{-L L_{1}^{2} \operatorname{sech} M \eta}{M}\right) \\
& +\beta\left(4 L^{2} L_{t} \operatorname{sech}^{2} M \eta\right) \\
& a_{18}=\lambda_{1}\left(\lambda_{1}-\lambda_{2}\right)\left(-3 L L_{1}^{2} \operatorname{sech} M \eta\right) \text {, } \\
& a_{19}=\lambda_{1}\left(\lambda_{1}-\lambda_{2}\right)\left(\frac{4 L L_{1}^{2} \operatorname{sech} M \eta}{M}-5 L^{2} L_{t} \operatorname{sech}^{2} M \eta\right) \text {, } \\
& a_{20}=\lambda_{1}\left(\lambda_{1}-\lambda_{2}\right)\left(3 L L_{1} L_{t} M^{2} \operatorname{sech} M \eta\right) \text {, } \\
& a_{21}=\lambda_{1}\left(\lambda_{1}-\lambda_{2}\right)\left(-\frac{2 L^{2} L_{t} \operatorname{sech} M \eta}{M}-L^{2} L_{t t} \operatorname{sech}^{2} M \eta\right) \\
& -\beta\left(L L_{1} M \operatorname{sech} M \eta\right) \text {, } \\
& a_{22}=\lambda_{1}\left(\lambda_{1}-\lambda_{2}\right)\left(\frac{L^{2} L_{t}}{M}+2 L^{2} L_{t t} \operatorname{sech} M \eta\right) \\
& +\beta\left(L L_{t} M^{2} \operatorname{sech} M \eta\right) \\
& a_{23}=\lambda_{1}\left(\lambda_{1}-\lambda_{2}\right)\left(4 L^{2} L_{t} \operatorname{sech}^{2} M \eta\right) \text {, } \\
& a_{24}=4 \beta\left(\lambda_{1}-\lambda_{2}\right)\left(L M L_{1} \operatorname{sech} M \eta\right), \\
& a_{25}=-\left(\lambda_{1}-\lambda_{2}\right)\left(-L_{t} L_{3} M^{3}\right), \\
& a_{26}=-\lambda_{1}\left(\lambda_{1}-\lambda_{2}\right)\left(L^{2} L_{t t}\right) \text {, } \\
& a_{27}=\left(\lambda_{1}-\lambda_{2}\right)\left(4 L M^{3} L_{3} \operatorname{sech} M \eta\right), \\
& a_{28}=4\left(\lambda_{1}-\lambda_{2}\right) \\
& \times\left(2 M L_{2} \operatorname{sech} M \eta\right. \\
& \left.+A_{4} M^{2} \operatorname{sech} M \eta+2 L_{3} \operatorname{sech} M \eta\right) L M \\
& +6 \lambda_{1} \lambda_{2}(1+L) L L_{1} M \operatorname{sech} M \eta \\
& -6 \lambda_{1}^{2}(1+L) L L_{1} M \operatorname{sech} M \eta \\
& -6 \beta(1+L) L L_{1} M \operatorname{sech} M \eta \text {, } \\
& a_{29}=4\left(\lambda_{1}-\lambda_{2}\right)\left(M L_{2}+4 L_{3}\right) L M^{2} \operatorname{sech} M \eta \\
& -6 \lambda_{1} \lambda_{2} L^{2} L_{t} M^{3} \operatorname{sech}^{2} M \eta \\
& +6 \lambda_{1}^{2} L^{2} L_{t} M^{3} \operatorname{sech}^{2} M \eta \\
& +6 \beta L^{2} L_{t} M^{3} \operatorname{sech}^{2} M \eta \\
& a_{30}=-2\left(2 L_{3 t}+L_{2 t} M\right) \text {, } \\
& a_{31}=-2\left(L_{2 t}+\frac{L_{1}}{M}-A_{4 t} M\right) \text {, } \\
& a_{32}=-2\left(A_{2 t}-L_{t}\right), \quad a_{33}=-M\left(3 a_{17}+2 a_{19}\right) \text {, } \\
& a_{34}=R\left(\frac{L_{1} L_{3}}{M}-L L_{3 t} \operatorname{sech} M \eta\right), \\
& a_{35}=-a_{29 x}-2\left(a_{12}+a_{15}\right) M \text {, } \\
& a_{36}=-2\left(a_{21}+a_{24}\right) M, \\
& a_{37}=-\left(2 a_{18}+3 a_{23}\right) M \text {, } \\
& a_{38}=-\left(a_{25}+L_{t} L_{3} M \operatorname{Re}\right) M \text {, } \\
& a_{39}=-\left(2 a_{13}+a_{30}\right) \\
& -\left(a_{16}+a_{20}\right) M+2 L_{3 t} \operatorname{Re}(1+L) \\
& \text { - } L_{t} M \operatorname{Re}\left(L_{2}+A_{4} M\right) A_{2 t} L M R \operatorname{sech} M \eta \\
& +L_{2 t} M R(1+L) \text {, } \\
& a_{40}=-\left(a_{12}+a_{28 x}+a_{14} M\right) \text {, } \\
& a_{41}=-\left(a_{15}+a_{14} M\right)+\left(3 L_{3}+L_{2}\right) \frac{L_{1} R}{M} \\
& -\left(L_{2 t}+\frac{L_{3 t}}{M}\right) L R \operatorname{sech} M \eta, \\
& a_{42}=-3 a_{25}-a_{13} M \\
& +2 L_{31 x} M-\left(3 L_{t} L_{3}-L_{3 t}\right) M R \\
& +\left(L L_{3 t}-L_{t} L_{2} M\right) M R \\
& a_{43}=-\left(a_{16}+a_{20}+a_{31 x}\right)+\frac{1}{M}\left(L_{1 t}-A_{2} L_{1} R\right) \\
& -\left(a_{11}-A_{4 t}(1+L)-A_{4} L_{t} R\right) M \\
& +\left(L_{t} L_{2}+(1+L) L_{2 t}-A_{2 t} L R \operatorname{sech} M \eta\right) R \text {, } \\
& a_{44}=\frac{-L_{1} L_{3} R}{2 M^{2}}+\frac{L L_{3 t} R \operatorname{sech} M \eta}{2 M}, \\
& a_{45}=-L_{1} L_{3} \operatorname{Re}-L L_{3 t} M R \operatorname{sech} M \eta \text {, } \\
& a_{46}=-\frac{a_{19}}{12}+\frac{a_{33}}{12 M}, \\
& a_{47}=-\frac{1}{8 M^{2}}\left(a_{27 x}+a_{35}\right)+\frac{1}{4 M}\left(a_{40}+a_{41}+2 a_{44}\right) \text {, }
\end{aligned}
$$




$$
\begin{aligned}
& a_{48}=-\frac{3 a_{38}}{M^{2}}+\frac{a_{42}}{M}, \\
& a_{49}=-\frac{3 a_{19}}{4}-\frac{1}{M^{4}}\left(2 M a_{42}+6 a_{38}\right) \\
& -\frac{1}{4 M}\left(a_{33}+4 a_{43}\right) \text {, } \\
& a_{50}=-\frac{a_{18}}{12}+\frac{a_{37}}{12 M}, \quad a_{51}=\frac{a_{27 x}}{4 M^{2}}+\frac{a_{34}}{2 M}+\frac{a_{35}}{4 M}, \\
& a_{52}=\frac{6 a_{38}}{M^{3}}-\frac{2 a_{42}}{M^{2}}+\frac{a_{39}}{M}, \\
& a_{53}=\frac{3 a_{18}}{4}-a_{22}+\frac{a_{37}}{4 M}, \\
& a_{54}=\frac{a_{27 x}}{6}+\frac{a_{45}}{3}, \\
& a_{55}=-a_{32 x}-\frac{a_{40}}{2}+\frac{a_{41}}{2}-L_{t t}, \\
& b_{11}=-\left(3 a_{17}-2 a_{19}\right) M \\
& +6 \beta\left(4 L_{1}^{2}+L M L_{1 t} \operatorname{sech} M \eta\right) L \operatorname{sech} M \eta \\
& +6 \lambda_{1}\left(\lambda_{1}-\lambda_{2}\right) \\
& \times\left(4 L_{1}^{2}+L M L_{1 t} \operatorname{sech} M \eta\right) L \operatorname{sech} M \eta, \\
& b_{12}=\left(L_{1} L_{3}+L L_{3 t} M \operatorname{sech} M \eta\right) R \\
& +4\left(\lambda_{1}-\lambda_{2}\right) \\
& \times\left(L_{1} L_{3}+L L_{3 t} M \operatorname{sech} M \eta\right) M^{2}, \\
& b_{13}=-\left(a_{12}+M a_{14}-A_{4} L_{1} \operatorname{Re}\right) \\
& +\frac{2 L_{1} R}{M^{2}}\left(L_{3}+M L_{2}\right) \\
& -6 \beta\left((1+L) L_{1}^{2}\right. \\
& \left.-\left(L_{1} L_{t}-L_{1 t}(1+L)\right) L M \operatorname{sech} M \eta\right) \\
& +A_{4 t} L M R \operatorname{sech} M \eta+\left(\lambda_{1}-\lambda_{2}\right) \\
& \times\left(8 L_{1}\left(L_{3}+M L_{2}\right)+4 A_{4} L_{1} M^{2}(1+M \operatorname{sech} M \eta)\right. \\
& \left.+8 L\left(L_{3 t}+L_{2 t} M\right) M \operatorname{sech} M \eta\right) \\
& -6 \lambda_{1}\left(\lambda_{1}-\lambda_{2}\right) \\
& \times\left(L_{1}^{2}(1+L)+\left(L_{1} L_{t}+L_{1 t}(1+L)\right) L M \operatorname{sech} M \eta\right), \\
& b_{14}=-\left(a_{25}+L_{t} L_{3} M R\right) M, \\
& b_{15}=-\left(2 a_{18}+3 a_{23}\right) M+6 \beta L^{2} L_{1 t} M^{2} \operatorname{sech}^{2} M \eta \\
& +6 \lambda_{1}\left(\lambda_{1}-\lambda_{2}\right) L^{2} L_{1 t} M^{2} \operatorname{sech}^{2} M \eta, \\
& b_{16}=-2\left(a_{12}+a_{15}\right) M \\
& +2 \frac{R}{M}\left(L_{1} L_{3}-L L_{3 t} M \operatorname{sech} M \eta\right) \\
& \text { - } 12 \beta M^{2} L L_{1} L_{t} M^{2} \operatorname{sech} M \eta \\
& +4\left(\lambda_{1}-\lambda_{2}\right)
\end{aligned}
$$

$$
\begin{aligned}
& \times\left(\left(4 L_{3}+L_{2} M\right) L_{1} M\right. \\
& +\left(4 L_{3 t}+L_{2 t} M\right) L M \operatorname{sech} M \eta \\
& \left.-12 \lambda_{1}\left(\lambda_{1}-\lambda_{2}\right) L L_{1} L_{t} M^{2} \operatorname{sech} M \eta\right), \\
& b_{17}=-2\left(a_{21}+a_{24}\right) M \\
& -6 \beta L^{2} L_{t t} M^{3} \operatorname{sech}^{2} M \eta \\
& -6 \lambda_{1}\left(\lambda_{1}-\lambda_{2}\right) L^{2} L_{1 t} M^{3} \operatorname{sech}^{2} M \eta, \\
& b_{18}=-2\left(a_{13}-2 L_{31 x}\right) \\
& -\left(\left(a_{16}+a_{20}+2 L_{21 x}\right) M+2(1+L) L_{3 t}\right. \\
& +\left((1+L) L_{2 t}-L_{t} L_{2}-A_{4} L_{t} M\right. \\
& \left.\left.+A_{2 t} L \operatorname{sech} M \eta\right) M\right) R, \\
& b_{19}=-\left(3 a_{25}+M\left(a_{13}-2 L_{31 x}\right) M\right) \\
& +\left(L_{3 t}(1+L)-L_{t}\left(3 L_{3}+L_{2} M\right) M R\right), \\
& b_{20}=-\left(a_{16}+a_{20}-2 L_{21 x}\right)+M\left(2 A_{41 x}-a_{11}\right) \\
& +R\left(L_{t} L_{2}+L_{2 t}(1+L)\right. \\
& \left.-A_{2 t} L \operatorname{sech} M \eta-\frac{A_{2} L_{1}}{M}\right) \\
& +\left(A_{4 t}(1+L)+A_{4} L_{t}\right) M R, \\
& b_{21}=-\left(L_{1} L_{3}+L L_{3 t} M \operatorname{sech} M \eta\right) R, \\
& b_{22}=-\left(a_{15}+a_{14} M\right) \\
& \text { - }\left(A_{4} M L_{1}-L_{1} L_{2}\right. \\
& \left.-\left(L_{2 t}-A_{4 t} M\right) M L \operatorname{sech} M \eta\right) \frac{R}{M}, \\
& b_{23}=2 A_{21 x}-L_{t t}+\left(A_{2 t}(1+L)+A_{2} L_{t}\right) R, \\
& c_{1}=\left(\left(b_{11}-a_{33}\right) \cosh M \eta\right. \\
& \left.+\left(b_{12}+a_{27 x}\right) \eta^{2}-a_{40}\right) \sinh ^{2} M \eta \\
& +\left(b_{21} \eta^{2}+b_{22}-a_{41}\right) \cosh ^{2} M \eta \\
& \times\left(\left(b_{14}-a_{38}\right) \eta^{2}\right. \\
& +\left(\left(b_{15}-a_{37}\right) \cosh M \eta\right. \\
& \left.+\left(b_{16}-a_{35}\right) \eta+b_{17}-a_{36}\right) \cosh M \eta \\
& \left.+\left(b_{18}+a_{39}\right) \eta\right) \sinh M \eta \\
& +\left(\left(b_{19}-a_{42}\right) \eta^{2}+b_{20}-a_{43}\right) \cosh M \eta-a_{44} \cosh 2 M \eta \\
& -a_{34} \eta \sinh 2 M \eta-a_{45} \eta^{2}+L_{2}+b_{23}+a_{23 x} \text {, } \\
& c_{3}=\frac{1}{24 M^{3}}(\operatorname{cosch} M \eta \\
& \times\left(12 a_{53} M^{2} \eta \cosh M \eta\right. \\
& +6 a_{53} M \sinh M \eta+4 a_{36} \sinh 2 M \eta \\
& \left.\left.+9 a_{50} M \sinh 3 M \eta\right)\right) \text {, }
\end{aligned}
$$




$$
\begin{aligned}
& c_{4}=\frac{1}{432 M^{6}} \\
& \times(432 \operatorname{sech} M \eta \\
& \times\left(-6 a_{54} M-M^{3}\left(a_{55}+c_{1}+3 a_{54} \eta^{2}\right)\right) \\
& -\left(81 a_{38}-54 M^{2}\right. \\
& \times\left(a_{48}-a_{52} M+4 a_{49} M^{2}-\eta^{2}\right. \\
& \times\left(3 a_{38}-M^{2}\left(2 a_{48}+2 a_{52} M\right.\right. \\
& \left.\left.\left.\left.+a_{38} \eta^{2}\right)\right)\right)\right) \cosh M \eta \\
& -M\left(112 a_{27 x}\right. \\
& +M^{2}\left(240 a_{51}-288 a_{47} M\right. \\
& \left.\left.+72 a_{27 x} \eta^{2}\right)\right) \cosh 2 M \eta \\
& +162 a_{46} M^{4} \cosh 3 M \eta \\
& +M \eta\left(162 a_{38}\right. \\
& -108 M^{2} \\
& \times\left(a_{48}-a_{52} M-2 a_{49} M^{2}\right. \\
& \left.\left.-\eta^{2}\left(a_{38}+\frac{2}{3} a_{48} M^{2}\right)\right)\right) \sinh M \eta \\
& +24 M^{2} \eta\left(5 a_{27 x}+12 a_{51} M^{2}\right) \sinh 2 M \eta \text {. }
\end{aligned}
$$

\section{Conflict of Interests}

The authors declare that there is no conflict of interests regarding the publication of this paper.

\section{Acknowledgments}

The authors are grateful to the reviewers for their valuable suggestions which improved the quality of the paper.

\section{References}

[1] A. H. Shapiro, M. Y. Jaffrin, and S. L. Weinberg, "Peristaltic pumping with long wave length at low Reynolds number," Journal of Fluid Mechanics, vol. 37, no. 4, pp. 799-825, 1969.

[2] A. Ebaid, "Effects of magnetic field and wall slip conditions on the peristaltic transport of a Newtonian fluid in an asymmetric channel," Physics Letters A, vol. 372, no. 24, pp. 4493-4499, 2008.

[3] Y. C. Fung and C. S. Yih, "Peristaltic transport," Journal of Applied Mechanics, vol. 33, pp. 669-675, 1968.

[4] T. Hayat, N. Ali, and S. Asghar, "An analysis of peristaltic transport for flow of a Jeffrey fluid," Acta Mechanica, vol. 193, no. 1-2, pp. 101-112, 2007.

[5] M. H. Haroun, "Effect of relaxation and retardation time on peristaltic transport of the Oldroydian viscoelastic fluid,"
Journal of Applied Mechanics and Technical Physics, vol. 46, no. 6, pp. 842-850, 2005.

[6] T. Hayat and N. Ali, "Peristaltic mechanism of a Maxwell fluid in an asymmetric channel," Nonlinear Analysis: Real World Applications, vol. 9, no. 4, pp. 1474-1490, 2008.

[7] S. Srinivas, R. Gayathri, and M. Kothandapani, “The influence of slip conditions, wall properties and heat transfer on MHD peristaltic transport," Computer Physics Communications, vol. 180, no. 11, pp. 2115-2122, 2009.

[8] T. K. Mittra and S. N. Prasad, "On the influence of wall properties and Poiseuille flow in peristalsis," Journal of Biomechanics, vol. 6, no. 6, pp. 681-693, 1973.

[9] M. A. Muthu, K. Rathish, and C. Peeyush, "Peristaltic motion in circular cylindrical tubes: effect of wall properties," Indian Journal of Pure and Applied Mathematics, vol. 32, no. 9, pp. 13171325, 2001.

[10] M. A. Abd Elnaby and M. H. Haroun, "A new model for study the effect of wall properties on peristaltic transport of a viscous fluid," Communications in Nonlinear Science and Numerical Simulation, vol. 13, no. 4, pp. 752-762, 2008.

[11] N. Ali, T. Hayat, and S. Asghar, "Peristaltic flow of a Maxwell fluid in a channel with compliant walls," Chaos, Solitons and Fractals, vol. 39, no. 1, pp. 407-416, 2009.

[12] T. Hayat, M. Javed, and N. Ali, "MHD peristaltic transport of a Jeffery fluid in a channel with compliant walls and porous space," Transport in Porous Media, vol. 74, no. 3, pp. 259-274, 2008.

[13] T. Hayat, S. Hina, and A. A. Hendi, "Heat and mass transfer effects on peristaltic flow of an Oldroyd-B fluid in a channel with compliant walls," Heat Transfer-Asian Research, vol. 41, no. 1, pp. 63-83, 2012.

[14] J. M. Burgers, "Mechanical considerations-model systemsphenomenological theories of relaxation and of viscosity," in First Report on Viscosity and Plasticity, Nordemann Publishing Company, New York, NY, USA, 1935.

[15] A. H. Gerritsen, C. A. P. M. van Gurp, J. P. J. van der Heide, A. A. A. Molenaar, and A. C. Pronk, "Prediction and prevention of surface cracking in asphaltic pavements," in Proceeding of the 6th International Conference on Structural Design of Asphalt Pavements (ISAP '87), vol. 1, University of Michigan, Ann Arbor, Mich, USA, July 1987.

[16] P. N. Chopra, "High-temperature transient creep in olivine rocks," Tectonophysics, vol. 279, no. 1-4, pp. 93-111, 1997.

[17] B. H. Tan, I. Jackson, and J. D. F. Gerald, "High-temperature viscoelasticity of fine-grained polycrystalline olivine," Physics and Chemistry of Minerals, vol. 28, no. 9, pp. 641-664, 2001. 


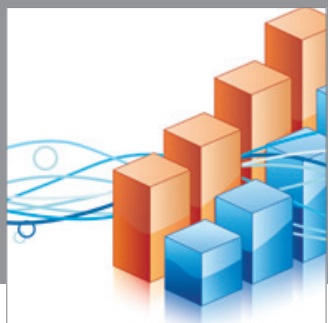

Advances in

Operations Research

mansans

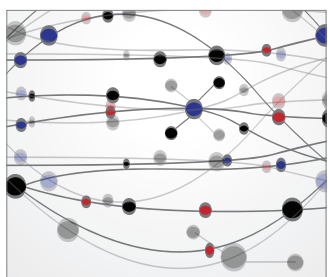

The Scientific World Journal
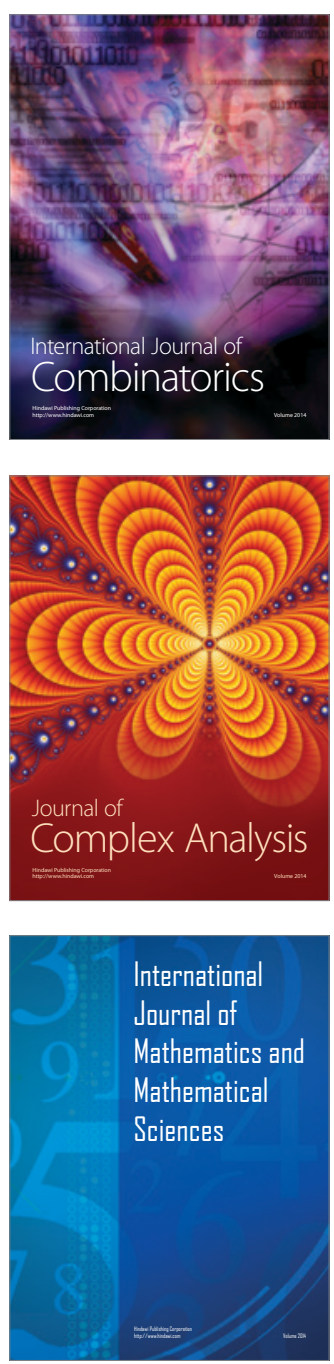
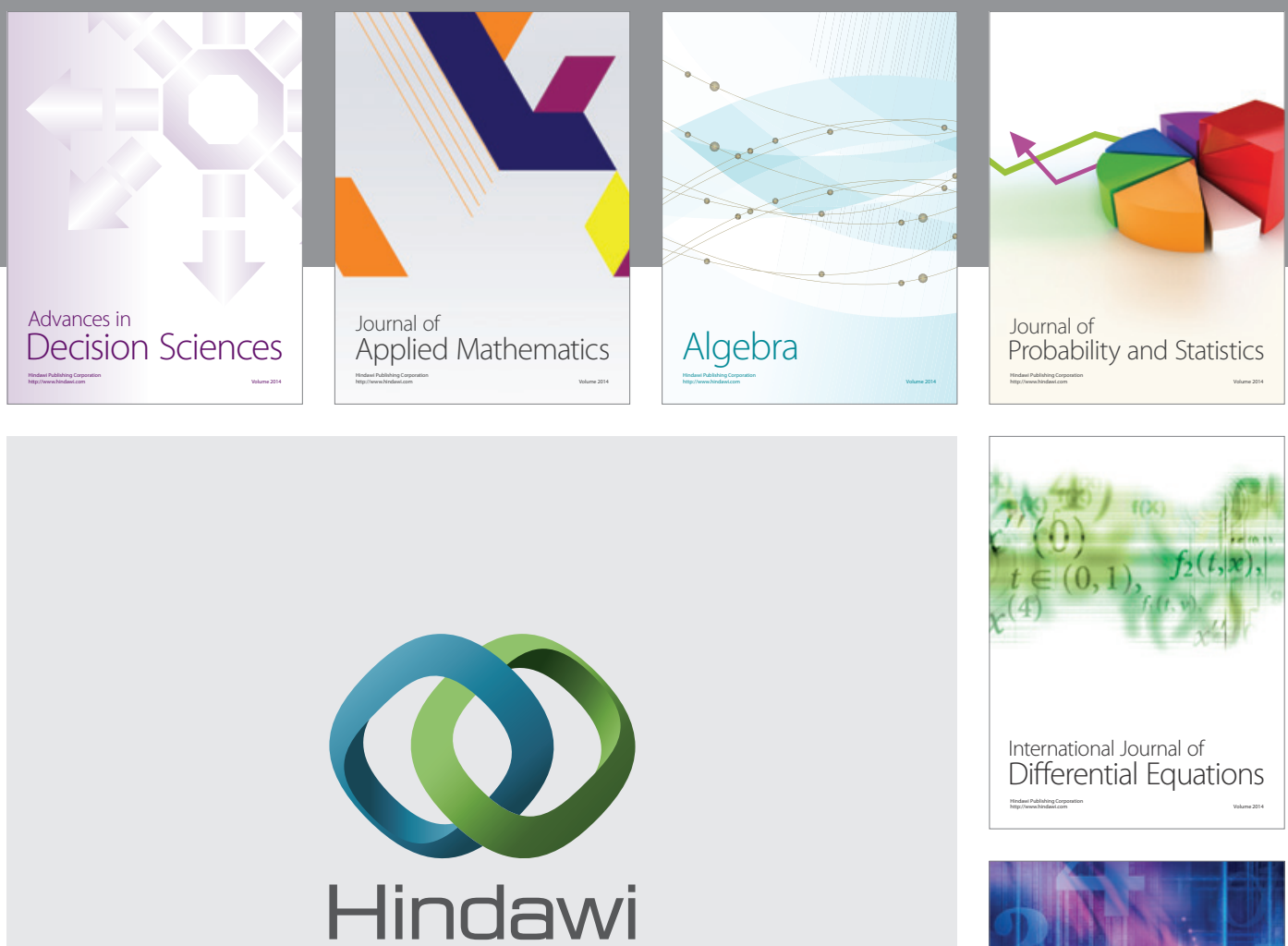

Submit your manuscripts at http://www.hindawi.com
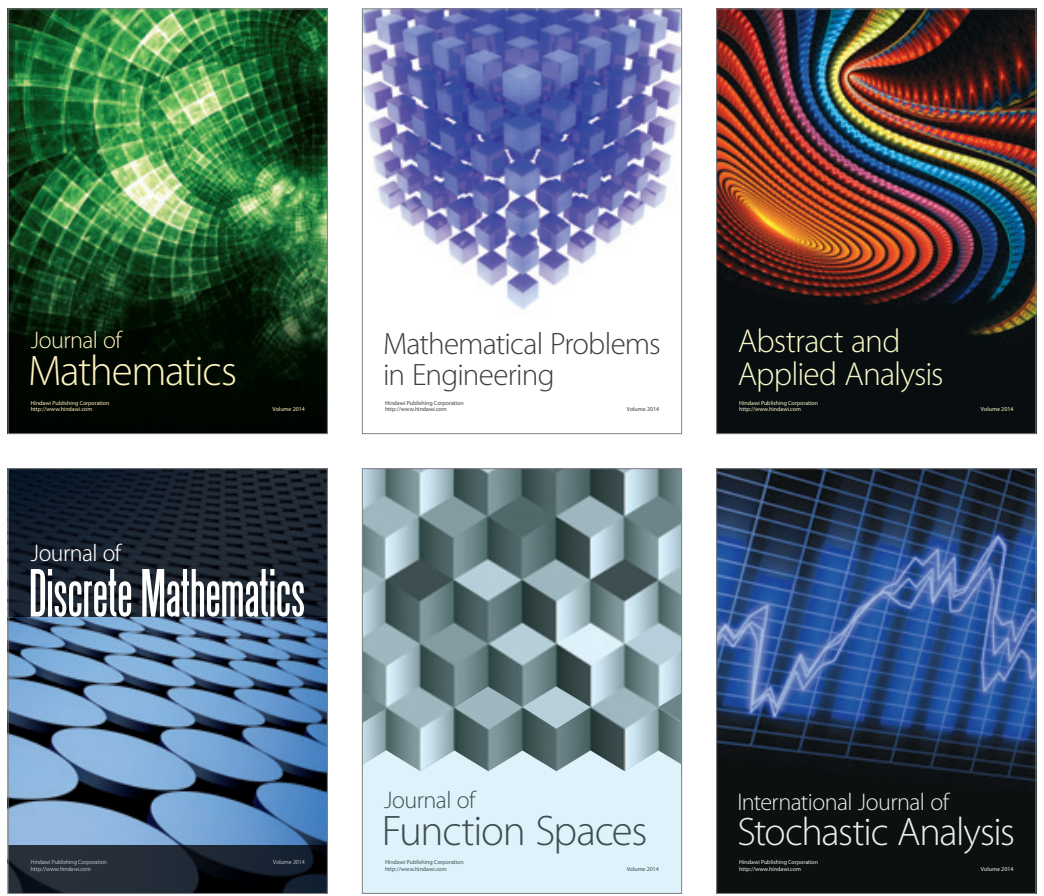

Journal of

Function Spaces

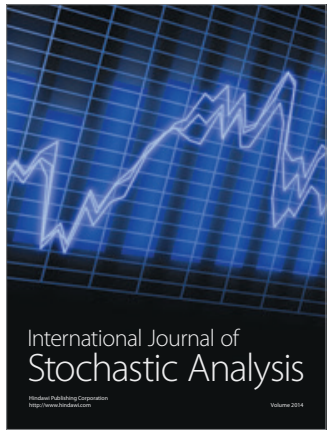

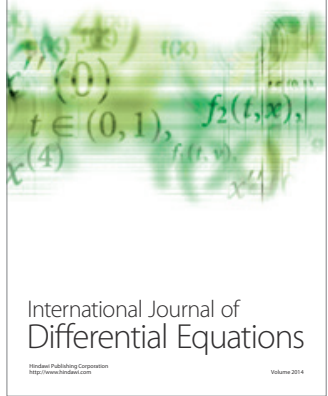
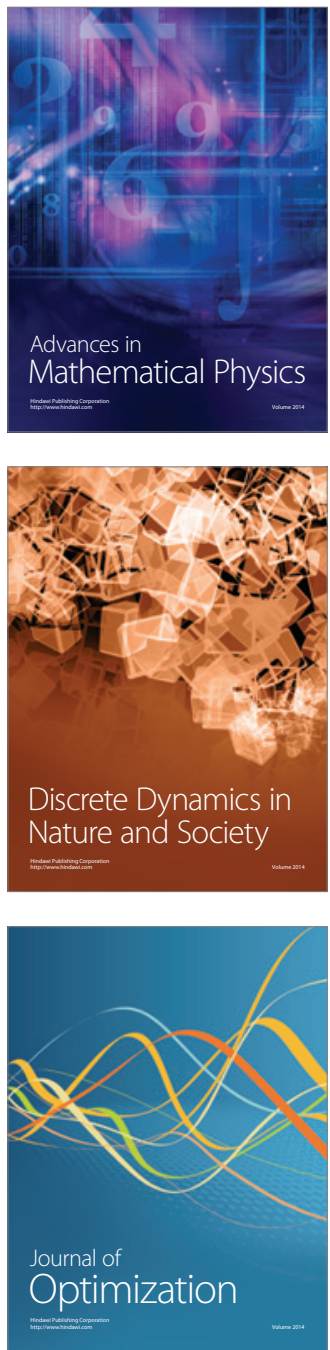